\title{
Combined application of pharyngeal volume and minimal cross-sectional area may be helpful in screening persons suspected of obstructive sleep apnea (OSA)
}

\author{
Yuliang Zhao ${ }^{1} \cdot$ Xinyu $\mathrm{Li}^{1} \cdot$ Jiangang Ma ${ }^{1}$ \\ Received: 25 December 2019 / Revised: 17 September 2020 / Accepted: 24 March 2021 / Published online: 8 May 2021 \\ (C) The Author(s) 2021
}

\begin{abstract}
Background Obstructive sleep apnea (OSA) is a common disease that seriously affects human health and daily life. However, the gold standard for its diagnosis, polysomnography (PSG), is expensive resulting in inadequate diagnosis of this disease in primary clinics. Therefore, a simple and rapid method for initial screening for OSA is needed. Acoustic pharyngometry (APh) is an FDAapproved noninvasive method that is gradually being applied to screening for OSA.

Materials And Methods In this study, we applied analysis with receiver operating characteristic (ROC) curves to explore how APh may play a greater role in the screening of subjects with suspected OSA. Patients admitted into the departments of otolaryngology at our hospital from March 2017 to May 2019 were recruited into the study. All subjects underwent PSG monitor and were separated into two groups according to the apnea-hypopnea index (AHI) from the PSG results: OSA group (AHI $\geq 5$ ) and control group $(\mathrm{AHI}<5)$. APh measurements and other indicators of the subjects, including age, height, and weight; Epworth Sleepiness Scale (ESS) score; and the pharynx examination, including the degree of tonsil enlargement and tongue hypertrophy, were also be recorded.

Results The $t$-test results showed that almost all indicators except age and height have significant differences between the OSA group and control group. Subjects with OSA had greater weight, BMI, ESS, higher degree of tonsil enlargement, and tongue hypertrophy, while they had smaller minimal cross-sectional area (mCSA) and pharyngeal volume than the subjects in control group. The correlation analysis revealed that pharyngeal volume and mCSA were two helpful indicators to screen for OSA. Furthermore, we established the ROC curve and calculated the combining predictors (combining predictors = pharyngeal volume $+\mathrm{mCSA} *(-2.347) /(-0.225))$. The area under the ROC curve (AUC) of combining predictors was 0.917 (95\% CI 0.842-0.991, $P<0.001)$, which was higher than combinations of other two independent indicators. The cutoff point of combining predictors was found to be 59.84 (AUC $=0.917$, sensitivity $=0.80,1$-specificity $=0.06, P<0.001$ ).

Conclusions These findings suggest that $\mathrm{APh}$ is a simple, rapid, and economical detection method which may be useful in screening for OSA, especially in communities and primary clinics where PSG cannot be performed.
\end{abstract}

Keywords Obstructive sleep apnea (OSA) - Acoustic pharyngometry (APh) $\cdot$ Receiver operating characteristic (ROC) curve * Minimum cross-sectional area of pharyngeal cavity (mCSA) $\cdot$ Pharyngeal volume

\section{Background}

Obstructive sleep apnea (OSA) refers to repeated episodes of apnea and hypopnea, hypercapnia, and sleep disruption, causing a series of clinical syndromes of pathophysiological

Jiangang Ma

edisondean@aliyun.com

1 Department of Otolaryngology, The second hospital of Hebei Medical University, Shijiazhuang 050000, China changes [1]. Numerous studies have shown that OSA is associated with a variety of diseases, including hypertension [2]; coronary heart disease [3]; arrhythmia [4]; cerebrovascular disease [5]; type 2 diabetes [6]; nonalcoholic fatty liver disease [7]; kidney damage [8]; glaucoma [9]; sexual dysfunction [10]; and many other organ, multi-system damage. In order to effectively prevent the symptoms and these complications, a primary task is to screen for OSA in an early period.

In China, the diagnostic criteria for OSA was clearly defined at the 2002 Hangzhou Conference [11], and was revised in 2011 [12] and 2019 [13]. The current gold standard for the 
diagnosis and severity of OSA is overnight polysomnography (PSG). But the equipment for PSG monitoring is expensive and requires specialized venues and analysts. PSG cannot be universally utilized in community hospitals and primary clinics. Other examinations, such as Muller's test lack simple and objective quantitative indicators for judging the location and severity of the obstruction. These factors have greatly limited the early diagnosis and treatment of OSA.

Acoustic pharyngometry (APh) is a FDA-approved, noninvasive method used in sleep apnea clinics and researches [14]. As an emerging technique for measuring the volume and cross-sectional area of the pharyngeal cavity by the principle of acoustic reflection, $\mathrm{APh}$ is characterized by simplicity, rapidity, and noninvasiveness, making it widely used in the preliminary screening of sleep apnea. Previous studies about the pharyngeal detection with $\mathrm{APh}$ were almost for normal people $[14,15]$, and a study exploring the relationship between $\mathrm{APh}$ and OSA did not clearly point out specific indicators and reference values [16].

Receiver operating characteristic (ROC) curve analysis is widely used as an estimate of the diagnostic value for many diseases. Based on our previous study on the application of $\mathrm{APh}[17,18]$, we selected and measured the APh parameters of the subjects. Combined with PSG monitoring results, ESS score, and anthropometric variables, we tried to explore the upper airway anatomy of patients with OSA, and seek the correlations between APh parameters and the severity of OSA. The purpose of this investigation was to assess whether or not it is possible to screen for OSA through measurement of pharyngeal volume and minimal cross-sectional area with acoustic pharyngometry.We proposed to use ROC analysis, to provide a more accurate analysis.

\section{Materials and methods}

\section{Protocol}

This study selected person who were admitted to the Department of Otolaryngology in our hospital from May 2017 to May 2019 as subjects.

\section{Inclusion criteria}

A. Age 18-65 years old.

B. No gender limitation.

C. The patient was initially determined to be suspected of OSA by the chief physician.

\section{Exclusion criteria}

A. Age $<18$ years old or $>65$ years old.
B. Nasopharyngeal or oropharynx diseases and other malformations.

C. Severe cardiopulmonary disease.

D. History of acute upper respiratory tract infection in the past month: nasal congestion, sneezing, salivation, etc.

E. Did not obtain the consent of the subject.

All of the subjects underwent PSG monitoring and were subsequently separated into two groups according to the apnea-hypopnea index (AHI): OSA group (AHI $\geq 5$ ) and control group $(\mathrm{AHI}<5)$. The $\mathrm{APh}$ measurement and other indicators of the subjects, including age, height, and weight; ESS score; pharynx examination; degree of tonsil enlargement; and tongue hypertrophy, were also recorded soon afterwards.

This study was approved by the Research Ethics Committee of the Second Hospital of Hebei Medical University. The approval number is 2018-R251.

\section{General information}

General information about the subjects such as gender and age was recorded. Height and weight of all subjects were measured and BMI was calculated $\left(\mathrm{BMI}=\right.$ height $/$ weight $\left.^{2}\right)$. The subjects also completed the ESS. Physical examination of the pharyngeal cavity structures was performed, the degree of tonsil enlargement and tongue hypertrophy (0structure, $\mathrm{d}$ and which is recorded as $0,1,2,3$ points, were recorded as described in previous studies $[19,20]$.

\section{Polysomnography monitor}

The Alice 4 polysomnography instrument (manufacturer: Compumedics; address: 30-40 Flockhart St. Abbotsford, Victoria, 3067, Australia) was used to perform night sleep monitoring for no less than $7 \mathrm{~h}$. The monitoring items included electroencephalogram, electrocardiogram, electromyogram, electrooculogram, saturation of blood oxygen $\left(\mathrm{SaO}_{2}\right)$, mouth and nasal airflow, chest and abdomen movement, position signal, and snoring. The results of the monitoring were analyzed by a single physician to obtain precise results. According to the criteria published by "Chinese Medicine Doctors Association Sleep medicine Specialized Committee (CMDASM) [13] and "American Academy of Sleep Medicine (AASM)" [21], "apnea" was defined as the nasal pressure signal 90\% lower than the baseline value, with duration not less than $10 \mathrm{~s}$. Hypopnea was scored when the nasal pressure signal was $50 \%$ lower than the baseline value, and the duration was not less than $10 \mathrm{~s}$, accompanied by $\mathrm{SaO}_{2}$ decrease of $3 \%$. AHI was calculated as the sum of the number of apneas and hypopneas that occurred per hour of sleep. 


\section{Acoustic pharyngometry}

An acoustic rhinometer instrument (manufacturer: GM INSTRUMENTS LTD; address: Unit 6, Ashgrove, Ashgrove Road Kilwinning, KA13 6PU, UK; name and version of the software: Acoustic, 3.2.0.1300) was used for $\mathrm{APh}$ measurement. All operations were performed by a single experienced physician. The measurement was carried out in a quiet room. The subject first assumed a sitting posture, tightened the sonic tube with the lips, and fixed it at the upper middle incisor to prevent air leakage keeping the sonic tube in a horizontal position (not tilted). The subject breathed through the nose 2 to 3 times and held his/her breath; then, he/she started the device for about 4-5 s. The sounder emitted 4 event waves, and the computer drew 4 curves. The measurement results were recorded in the form of a graph and obtained the area-distance curve that is the APh curve. The same method was used to measure the results in the supine posture and the lateral posture. Each operation was repeated 3 times, and the repetition rates were controlled within $10 \%$. The average values were taken to obtain a series of parameter values.

The $0 \mathrm{~cm}$ of the abscissa of the APh curve corresponded to the upper middle incisor. The measurement range was 0-20 $\mathrm{cm}$, and the observation range was about $7-17 \mathrm{~cm}$, i.e. the oropharynx junction to the glottis area. The computer automatically calculated the volume of the area as the pharyngeal volume. According to the results of previous studies [17], the mCSA and the pharyngeal volume were selected as the parameters of APh in three postures (sitting posture, supine posture, and lateral posture).

\section{Statistical analyses}

Data were analyzed using IBM SPSS Statistics 25 software package. Continuous variables were expressed as mean \pm standard deviation. Student's $t$ test was used for continuous variables including age, height, weight, BMI, ESS score, AHI, $\mathrm{LSaO} 2$, SIT90, pharyngeal volume, and mCSA. Chi-square tests were used for discrete variables including gender. Kruskal-Wallis test was used for degree of tonsil enlargement and tongue hypertrophy. Correlation analysis was used to analyze the correlations between the pharyngeal volume and indicators of the PSG results (AHI, SIT90, and $\mathrm{LSaO}_{2}$ ) respectively. A $p$ value $<0.05$ was considered statistically significant. The ROC curve was established, the regression equation was obtained, the joint prediction factor was calculated, and the maximum Youden index as the cutoff point was selected. All illustrations were generated using Graph Prism 6.0 software.

\section{Result}

A total of 68 subjects were studied. There were 52 subjects in the OSA group, including 35 men and 17 women. There were 16 subjects in the control (non-OSA) group, including 12 men and 4 women. Chi-square test showed no significant difference in sex ratio between the two groups $\left(\chi^{2}=0.339\right.$, $P=0.560)$.

\section{Comparison of different indicators between OSA group and control group}

The results show that (1) there were no significant differences in the gender, age, and height between the control group and the OSA group; (2) body weight, BMI, ESS score, AHI, SIT90, tonsil enlargement, and tongue hypertrophy in the OSA group were significantly greater than those in the control group; and (3) the $\mathrm{LSaO}_{2}$, pharyngeal volume, and mCSA in three postures in the OSA group were significantly lower than those of the control group. Data are shown in Tables 1 and 2.

\section{Correlation analysis between APh results and PSG}

There were significant correlations between the pharyngeal volume vs. all indicators of the PSG results $(P<0.001)$. However, the correlations between the mCSA in the three postures vs. the PSG results were not exactly the same: There were significant correlations between the mCSA in the supine position vs. the indicators of the PSG results $(P<0.001, P=0.008, P=0.002)$; in the lateral posture, there were significant correlations between the mCSA vs. AHI $(P=0.006)$, while there was no significance vs. the other indicators; there was no significant correlation between the mCSA in the sitting vs. the indicators of the PSG results. Data shown are in Table 3.

\section{Established the ROC curve and acquired cutoff point}

According to the results above, we found that APh measurement of pharyngeal volume and $\mathrm{mCSA}$ in supine position were two favorable indicators for the screening of OSA. Next, we established the ROC curve to determine the effectiveness of these two indicators. The areas under the ROC curve (AUC) of pharyngeal volume and mCSA were 0.887 (95\% CI $0.789-0.985, P<0.001)$ and 0.828 (95\% CI $0.731-$ $0.925, P<0.001)$. Then, we calculated the combining predictors (a new indicator obtained by logistic regression analysis).

The regression equation was:

Logit $(Y)=13.750+(-0.225) *$ pharyngeal volume

$$
+(-2.347) * \mathrm{mCSA} \text {. }
$$


Table 1 Comparison of different indicators between OSA group and control group

\begin{tabular}{llllll}
\hline & Indicators & Control group & OSA group & $t$ & $P$ \\
\hline \multirow{2}{*}{ General condition } & Age & $35.5 \pm 2.6$ & $37.4 \pm 1.5$ & .6236 & 0.54 \\
& Height $(\mathrm{cm})$ & $171.9 \pm 1.6$ & $170.0 \pm 0.9$ & 1.006 & 0.32 \\
& Weight $(\mathrm{kg})$ & $68.8 \pm 2.3$ & $104.2 \pm 3.0$ & 6.245 & $<0.001$ \\
& BMI & $23.2 \pm 0.6$ & $36.0 \pm 1.0$ & 6.696 & $<0.001$ \\
& ESS & $1.9 \pm 0.4$ & $8.8 \pm 0.8$ & 4.587 & $<0.001$ \\
PSG results & AHI (times/h) & $0.6 \pm 1.1$ & $40.4 \pm 4.6$ & 4.419 & $<0.001$ \\
& LSaO $(\%)$ & $87 \pm 2$ & $72 \pm 2$ & 3.598 & 0.006 \\
& SIT90 & $0.36 \pm 0.24$ & $23.14 \pm 3.56$ & 3.527 & 0.008 \\
APh results & Pharyngeal volume $\left(\mathrm{cm}^{3}\right)$ & $48.6 \pm 1.2$ & $35.0 \pm 1.1$ & 6.650 & $<0.001$ \\
& mCSA (supine) $\left(\mathrm{cm}^{2}\right)$ & $1.77 \pm 0.06$ & $1.29 \pm 0.05$ & 4.786 & $<0.001$ \\
& mCSA (sitting) $\left(\mathrm{cm}^{2}\right)$ & $2.03 \pm 0.11$ & $1.70 \pm 0.06$ & 2.614 & 0.011 \\
& mCSA (lateral) $\left(\mathrm{cm}^{2}\right)$ & $1.68 \pm 0.11$ & $1.43 \pm 0.05$ & 2.300 & 0.025 \\
\hline
\end{tabular}

And the combining predictors were calculated as:

Combining predictors $=$ pharyngeal volume

$$
+\mathrm{mCSA} *(-2.347) /(-0.225) \text {. }
$$

The AUC of combining predictors was 0.917 (95\% CI 0.842-0.991, $P<0.001$ ), which was higher than other two independent indicators. The cutoff point of combining predictors was received as 59.84 ( $\mathrm{AUC}=0.917$, sensitivity $=0.80$, 1 -specificity $=0.06, P<0.001)($ Fig. 1$)$.

\section{Discussion}

\section{The primary general survey of OSA is imminent}

OSA is characterized by repeated episodes of apnea and hypopnea, hypercapnia, and sleep disruption. According to the World Health Organization, about 100 million people worldwide suffer from OSA [22]. A meta-analysis of OSA epidemiological studies [2] pointed out that the morbidity of OSA in the general adult population was 9-38\% worldwide and men's were slightly higher than women's. This proportion increases with age. In some elderly populations, the morbidity rate was as high as $90 \%$ for men and $78 \%$ for women [20].

Table 2 Kruskal-Wallis test for the degree of tonsil enlargement and tongue hypertrophy

\begin{tabular}{lllll}
\hline Indicators & \multicolumn{2}{l}{ Mean rank } & \multirow{2}{*}{$\chi^{2}$} & $P$ \\
\cline { 2 - 4 } & \multicolumn{1}{l}{ Control group } & OSA group & & \\
& & & \\
\hline Tonsil enlargement & 13.13 & 41.08 & 26.682 & $<0.001$ \\
Tongue hypertrophy & 19.25 & 39.19 & 13.350 & $<0.001$ \\
\hline
\end{tabular}

Some cross-sectional studies had reported a morbidity of OSA ranging from 40 to $90 \%$ in individuals with a BMI $>40 \mathrm{~kg} / \mathrm{m}^{2}$ (severe obesity) [23].

Although the known morbidity is high, the factual situation may be more serious. Young et al. estimated that up to $93 \%$ of women and $82 \%$ of moderate OSA patients have not been diagnosed; as many as one in five adults may have been mild undiagnosed OSA; more than one-tenth adults may had moderate undiagnosed disease [24-26]. The results of a selective screening study were startling: In the group only $19 \%$ of patients were previously diagnosed with OSA, but the diagnostic rate increased to $56 \%$ after being screened by clinical parameters and the ESS score. Furthermore, when all patients were examined by PSG, the diagnostic rate increased to $91 \%$ [27]. The underestimation of OSA diagnosis in the general population means there had been a large number of undiagnosed OSA patients who were mistaken for non-OSA patients. In terms of treatment, in the Sleep Heart Health Study of more than 15,000 people, the morbidity rate of OSA was more than $4 \%$, while only $1.6 \%$ of patients were diagnosed by doctors, and only $0.6 \%$ were actually treated [28]. These results indicated that the diagnosis of this disease was seriously

Table 3 Correlation analysis between APh results vs. PSG results

\begin{tabular}{lllll}
\hline Indicators & & AHI & SIT90 & $\mathrm{LSaO}_{2}$ \\
\hline Pharyngeal volume & Pearson correlation & -.690 & -.502 & .555 \\
& Sig. (two-tailed) & $<.001$ & $<.001$ & $<.001$ \\
mCSA (sitting) & Pearson correlation & -.231 & -.120 & .095 \\
& Sig. (two-tailed) & .058 & .328 & .440 \\
mCSA (supine) & Pearson correlation & -.525 & -.321 & .372 \\
& Sig. (two-tailed) & $<.001$ & .008 & .002 \\
mCSA (lateral) & Pearson correlation & -.328 & -.206 & .183 \\
& Sig. (two-tailed) & .006 & .091 & .135 \\
\hline
\end{tabular}


Fig. 1 The ROC curve of pharyngeal volume, mCSA, and combining predictors

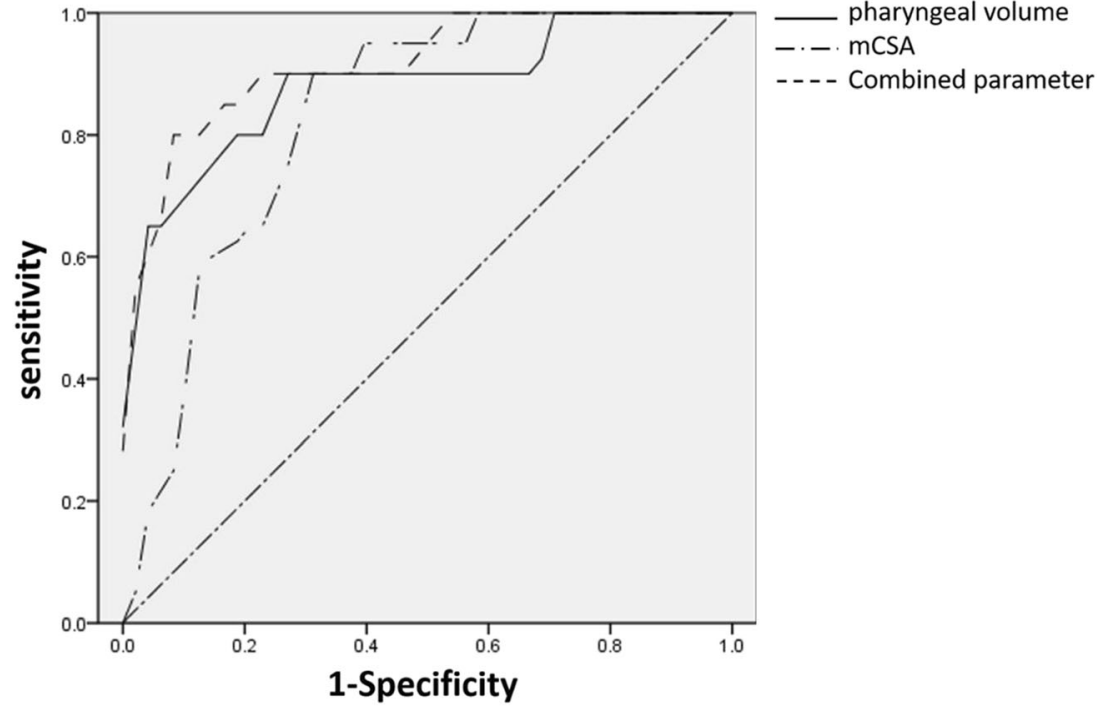

inadequate, and the general population has insufficient understanding of the severity of the disease and lack of willingness to be treated. Therefore, focusing on high-risk factors, getting patients identified sooner, and starting intervention sooner were important approaches to reducing the harms of OSA. The major reason for underdiagnosis is the limited diagnostic method. The gold standard is PSG, but the equipment is expensive and requires specialized venues and analysts. There are many other diagnostic methods for OSA described in the literature, but each has limitations. Neck circumference is easy to measure but underestimates OSA in lean individuals [29], and does not perform as well as pharyngometry. CT involves ionizing radiation exposure and is not readily available in many sleep clinics or other offsite centers. CT is also time-consuming and costly [30]. MRI avoids ionizing radiation and provides excellent definition of parapharyngeal soft tissues [31], but is expensive and not readily available in many sleep clinics. ESS was often used for the initial screening of OSA, but because of its subjectivity, the study of the effectiveness and accuracy of the scale had different conclusions [32]. Other examinations such as Muller's test always lack simple and objective quantitative indicators for judging the location and severity of obstruction. These factors have greatly limited the early diagnosis and treatment of OSA. Therefore, we urgently need a simple, convenient, and economical method for screening OSA which can be used in clinics.

\section{APh can be used for initial screening of OSA}

As an emerging technique for measuring the volume and cross-sectional area of the pharyngeal cavity by the principle of acoustic reflection, APh is an FDA-approved noninvasive diagnostic test that is gradually gaining use in screening for OSA. The APh is a simple instrument yielding rapid results.

In patients with OSA there is the narrowing or blocking of the upper respiratory tract from the oropharynx to the hypopharynx during sleep, resulting in ineffective or absent breathing. Accurate understanding of the upper respiratory tract anatomy is the key to screening for OSA. The Starling resistor model has been proposed to explain the complex series of events that occur in patients with OSA during sleep (Fig. 2) [24]. Pharyngeal size, compliance, and the dynamic behavior of the upper airway have been considered important factors in the pathogenesis of OSA. So, the assessment of the precise narrowing site of the upper airway may not be only one of the keys in understanding the pathogenesis of this disorder but also in improving the management of this condition. APh has the potential to be a useful tool for localizing the possible site(s) of upper airway obstruction in cases of OSA.

In 1984, Rilive et al. [33] first applied APh to patients with OSA. By measuring the upper respiratory tract morphology, it was concluded that the average cross-sectional area of the pharyngeal cavity and the average glottal cross-sectional area in OSA was smaller than in patients without OSA. Kamal et al. [15] used special techniques during the examination to locate the oropharyngeal junction and the glottis to generate a mapped acoustic throat map, calculating the minimum pharyngeal area and glottal area. The studies' results showed that the APh can accurately determine the pharyngeal obstruction [14], and had been validated by computerized axial tomography (CT) [34] and magnetic resonance imaging (MRI) [35].

The APh results showed good repeatability and a low degree of variability in the measurement of the oral cavity and 
Fig. 2 Starling resistor model [24]

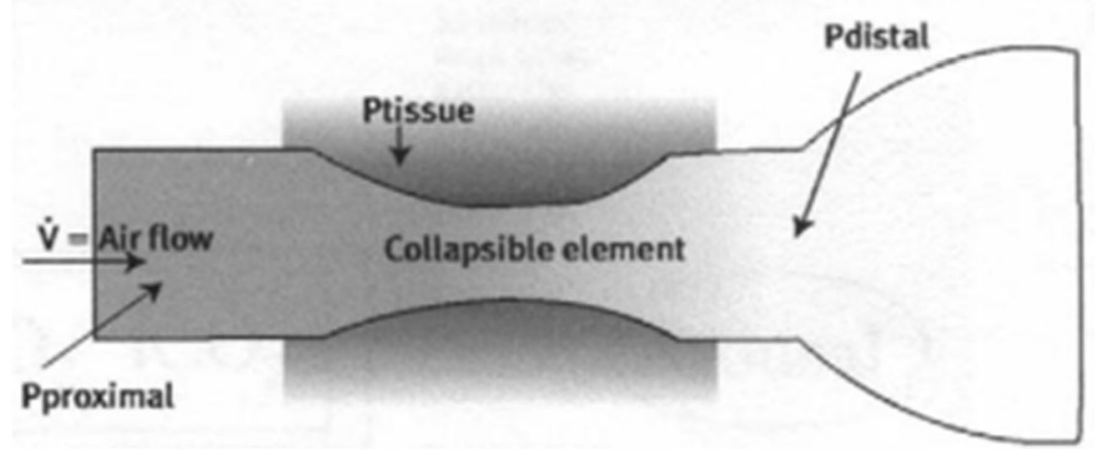

pharyngeal cavity [36]. In particular, mCSA obtained via APh has been established to have acceptable intrasession and testretest reliability [15].

\section{Combined application of pharyngeal volume and mCSA can be a favorable and helpful indicator of OSA screening}

The results of our study showed that subjects with OSA had higher weight and BMI than subjects with no OSA. Obesity has been considered to be a major risk factor for OSA and was closely related to the severity of OSA [37]. The upper airway structure of obese people is significantly different from normal people. With increased body fat and reduced lung volume (functional residual capacity), the upper airway in obese patients is narrower, and makes the airway vulnerable to collapse. Obesity can also lead to increased fat deposition around the pharyngeal wall and lymphoid tissue proliferation in the neck. The fat deposition in the pharynx has an external compression effect, which causes the pharyngeal cavity to change from a normal circular shape to an elliptical shape resulting in a reduction in the upper airway cross-sectional area and an increase in airflow resistance. Parts of the upper airway narrow (including nasal cavity, nasopharynx, oropharynx, and throat) affecting normal breathing [38]. Previous studies have shown that gender, age, height, and other factors affect the pharyngeal cavity structure (mainly pharyngeal volume) [39], and the mCSA decrease with age [40]. Examination of the upper airway has shown that subjects with OSA have more tonsil enlargement and tongue hypertrophy than controls. Gun's study showed that the cross-sectional area and volume of the upper airway is smaller in the supine position than any other positions and is the most predictive parameter to discriminate between subjects with or without OSA [41]. Another study demonstrated that Aph measurement of mCSA is a significant independent predictor of moderate-tosevere obstructive sleep apnea [42], which is consistent with our study. In all, a variety of factors such as obesity, inflammation, and metabolic disorders may cause complex changes in the structure of the pharyngeal cavity and lead to OSA.

\section{Applied ROC curve to conduct a further analysis of the APH results}

The main purpose of our study was to find the appropriate parameters using APh for indicating OSA. Based on previous studies [15] and the results of our investigation, we have found that pharyngeal volume and $\mathrm{mCSA}$ are useful to screen for suspected OSA when PSG is not available. According to the ROC curve analysis, combining predictors had the largest AUC. The findings of this research confirms that $\mathrm{APh}$ is a simple and rapid method to screen for OSA. Aph may be especially pertinent for use in communities and primary clinics where PSG cannot be performed.

\section{Conclusion}

The current study provides evidence that $\mathrm{APh}$ may play a role in screening for OSA. Pharyngeal volume and mCSA appear to be helpful indicators suggesting the presence of OSA. If combining predictors (calculated as: combining predictors $=$ pharyngeal volume $+\mathrm{mCSA} *(-2.347) /(-0.225))$ show a score higher than 59.84, OSA is very likely (sensitivity $=0.80$, 1 -specificity $=0.06$ ) and suggests the need for further examination with PSG.

Acknowledgements Thanks to everyone who has supported and helped this research, including other doctors, nurses, and graduate students in our department.

Author contribution Zhao conceived of the study, collected literature, performed the statistical analysis, and was a major contributor in writing the manuscript. Li collected patients' information and did all the examinations. Ma conceived of the study, did all the diagnosis and participated in its design and coordination, and helped to draft the manuscript. All authors read and approved the final manuscript.

Funding This study was supported by the "Hebei Province Science and Technology Support Program (no. 182777199)" and the "Department of Health of Hebei Province (CN) (no. 20190576)."

Data availability All data generated or analyzed during this study are included in this published article. The database during and/or analyzed 
during the current study available from the corresponding author on reasonable request.

\section{Declarations}

Ethics approval and consent to participate This study was approved by Research Ethics Committee of the second hospital Of Hebei Medical University. Based on the study design, after the review of the Ethics Committee, the health, right, and privacy of subjects are fully protected; potential risk and harm to the subjects are controllable to minimal. This study was approved by Research Ethics Committee of the second hospital of Hebei Medical University. The approval number is 2018-R251.

Consent for publication All presentations of data have consent for publication.

Competing interests The authors declare no competing interests.

Abbreviations OSA, obstructive sleep apnea; $\mathrm{APh}$, acoustic pharyngometry; ROC, receiver operating characteristic; ESS, Epworth Sleepiness Scale; mCSA, minimal cross-sectional area; PSG, polysomnography; $\mathrm{SaO}_{2}$, saturation of blood oxygen; $\mathrm{AHI}$, apnea and hypopnea index; $\mathrm{LSaO}_{2}$, lowest saturation of blood oxygen; SIT90\%, salvation impair time below $90 \%$; CT, computerized axial tomography; MRI, magnetic resonance imaging

Open Access This article is licensed under a Creative Commons Attribution 4.0 International License, which permits use, sharing, adaptation, distribution and reproduction in any medium or format, as long as you give appropriate credit to the original author(s) and the source, provide a link to the Creative Commons licence, and indicate if changes were made. The images or other third party material in this article are included in the article's Creative Commons licence, unless indicated otherwise in a credit line to the material. If material is not included in the article's Creative Commons licence and your intended use is not permitted by statutory regulation or exceeds the permitted use, you will need to obtain permission directly from the copyright holder. To view a copy of this licence, visit http://creativecommons.org/licenses/by/4.0/.

\section{References}

1. West SD, Turnbull C (2018) Obstructive sleep apnoea. Eye:32(5)

2. Senaratna CV, Perret JL, Lodge CJ, Lowe AJ, Campbell BE, Matheson MC, Hamilton GS, Dharmage SC (2016) Prevalence of obstructive sleep apnea in the general population: a systematic review. Sleep Med Rev 34:70

3. Xie C, Zhu R, Tian Y, Kai W (2017) Association of obstructive sleep apnoea with the risk of vascular outcomes and all-cause mortality: a meta-analysis. BMJ Open 7(12):e013983

4. Szymanski FM, Filipiak KJ, Platek AE, Hrynkiewiczszymanska A, Kotkowski M, Kozluk E, Kiliszek M, Sierdzinski J, Opolski G (2015) Presence and severity of obstructive sleep apnea and remote outcomes of atrial fibrillation ablations - a long-term prospective, cross-sectional cohort study. Sleep Breath 19(3):1-8

5. Johnson KG, Johnson DC (2010) Frequency of sleep apnea in stroke and TIA patients: a meta-analysis. J Clin Sleep Med 6(2): 131-137

6. Wang X, Bi Y, Zhang Q, Pan F (2013) Obstructive sleep apnoea and the risk of type 2 diabetes: a meta-analysis of prospective cohort studies. Respirology 18(1):140-146
7. Jin S, Jiang S, Hu A (2018) Association between obstructive sleep apnea and non-alcoholic fatty liver disease: a systematic review and meta-analysis. Sleep Breath 22(1):1-11

8. Chen Y, Li Y, Jiang Q, Xu X, Zhang X, Simayi Z, Ye H (2015) analysis of early kidney injury-related factors in patients with hypertension and obstructive sleep apnea hypopnea syndrome (OSAHS). Archives of Iranian Medicine 18(12):827-833

9. Pezrico C, Guti Jiang Q, Xu X, Zhang X, Simayi Z, Ye H (2014) Analysis of early kidney injury-related factors in patients with hypertension and obstructive sleep. 252(9): 1345

10. Campos-Juanatey F, Fernandez-Barriales M, Gonzalez M, PortilloMartin JA (2017) Effects of obstructive sleep apnea and its treatment over the erectile function: a systematic review. Asian Journal of Andrology 19(3):303-310

11. Chinese Medical Association of Otorhinolaryngology, Head and Neck Surgery (2002) Diagnostic criteria and efficacy evaluation criteria for obstructive sleep apnea hypopnea syndrome and indications for uvulopalatopharyngoplasty (Hangzhou). Chinese Journal of Otorhinolaryngology Head and Neck Surgery 37(6):403-404

12. Chinese Medical Association of Sleep Respiratory Disorders Group, Qingyun L (2012) Expert consensus on the clinical application of continuous positive airway pressure in patients with obstructive sleep apnea hypopnea syndrome. Chinese Journal of Tuberculosis and Respiratory Diseases 35(1):13-18

13. Chinese Medicine Doctors Association Sleep medicine Specialized Committee (CMDASM) (2018) Multidisciplinary guideline for diagnostic testing for adult obstructive sleep apnea. Chin Med J 2: 1902-1914

14. Molfenter SM (2016) The reliability of oral and pharyngeal dimensions captured with acoustic pharyngometry. Dysphagia 31(4):1-5

15. Kamal I (2001) Normal standard curve for acoustic pharyngometry. Otolaryngol Head Neck Surg 124(3):323-330

16. Gun JD, Young CH, Grunstein RR et al (2004) Predictive value of Kushida index and acoustic pharyngometry for the evaluation of upper airway in subjects with or without obstructive sleep apnea. J Korean Med Sci 19(5):662

17. Ting R, Ma J, Yuliang Z (2019) Effects of different posture pharyngeal reflexes on the assessment of OSAHS and its evaluation of the therapeutic effect of OSAHS. Journal of Otorhinolaryngology Head and Neck Surgery 33(2):118-122

18. Ting R, Ma J, Xinyu L, Li C, Xinxia J, Wang Y (2018) Progress in the application of pharyngeal reflex in the diagnosis and treatment of OSAHS. Journal of Otorhinolaryngology Head and Neck Surgery 6

19. Cassano P, Gelardi M, Cassano M, Fiorella ML, Fiorella R (2003) Adenoid tissue rhinopharyngeal obstruction grading based on fiberendoscopic findings: a novel approach to therapeutic management [J]. Int J Pediatr Otorhinolaryngol 67(12):1303-1309

20. Mlynarek A, Tewfik MA, Hagr A, Manoukian JJ, Schloss MD, Tewfik TL, Choi-Rosen J (2004) Lateral neck radiography versus direct video rhinoscopy in assessing adenoid size. J Otolaryngol 33(6):360-365

21. Kapur VK, Auckley DH, Chowdhuri S et al. (2017) Clinical practice guideline for diagnostic testing for adult obstructive sleep apnea: an American Academy of Sleep Medicine Clinical Practice Guideline. Journal of clinical sleep medicine: JCSM: official publication of the American Academy of Sleep Medicine, 13(3).

22. World Health Organization (2007) Global surveillance, prevention and control of chronic respiratory diseases: a comprehensive approach. World Health Organization, Geneva

23. Peppard PE, Young T et al. (2000) Longitudinal study of moderate weight change and sleep-disordered breathing, JAMA 284 30150) 3015

24. Moos DD, Cuddeford JD (2006) Implications of obstructive sleep apnea syndrome for the perianesthesia nurse. Journal of Perianesthesia Nursing 21(2):103-118 
25. Young T, Evans L, Finn L, Palta M (1997) Estimation of the clinically diagnosed proportion of sleep apnea syndrome in middleaged men and women. Sleep. 20:705-706

26. Young T, Peppard PE, Gottlieb DJ (2002) Epidemiology of obstructive sleep apnea: a population health perspective. Am J Respir Crit Care Med 165:1217-1239

27. H PT, S TA, S M, G K, R A, C C, J JJ (2007) Potentially lifethreatening sleep apnea is unrecognized without aggressive evaluation. Am J Surg 193(3):364-367

28. Oltmanns KM (2008) Abdominal fat and sleep apnea: the chicken or the egg?: Response to Pillar and Shehadeh. Diabetes Care 31(7): e61 author reply e62

29. Davies RJO, Stradling JR (1990) The relationship between neck circumference, radiographic pharyngeal anatomy, and the obstructive sleep apnoea syndrome [J]. Eur Respir J 3(5):509-514

30. Haponik E (1983) Computerized tomography in obstructive sleep apnea: correlation of airway size with physiology during sleep and wakefulness.[J]. Am.rev.respir.dis, 127

31. Schellenberg J, Maislin G, Schwab R (2000) Physical findings and the risk for obstructive sleep apnea. The importance of oropharyngeal structures. Am J Respir Crit Care Med 162(2):740-748

32. Rasheid S, Banasiak M, Gallagher SF, Lipska A, Kaba S, Ventimiglia D, Anderson WM, Murr MM (2003) Gastric bypass is an effective treatment for obstructive sleep apnea in patients with clinically significant obesity. Obes Surg 13(1):58-61

33. Rivlin J, Hoffstein V, Kalbfleisch J, Mcnicholas W, Zamel N, Bryan AC (1984) Upper airway morphology in patients with idiopathic obstructive sleep apnea. Am Rev Respir Dis 129(3):355

34. Yu C, Wang G, Fu C, Liu Y (2010) Acoustic rhinometry and acoustic pharyngometry in the modeling of upper airway based on CT scan. The 4th international conference on bioinformatics and biomedical engineering; 2010; p. 1-4

35. Fujimoto Y, Huang J, Fukunaga T, Kato R, Higashino M, Shinomiya S, Kitadate S, Takahara Y, Yamaya A, Saito M (2013) A three-microphone acoustic reflection technique using transmitted acoustic waves in the airway. J Appl Physiol 115(8): 1119-1125

36. Marshall I, Maran NJ, Martin S, Jan MA, Rimmington JE, Best JJ, Drummond GB, Douglas NJ (1993) Acoustic reflectometry for airway measurements in man: implementation and validation. Physiol Meas 14(2):157-169

37. Greenburg DL, Lettieri CJ, Eliasson AH (2009) Effects of surgical weight loss on measures of obstructive sleep apnea: a meta-analysis. Am J Med 122(6):535-542

38. Mutairi SA, Mojiminiyi OA, Alawi AA, Rammah TA, Abdella N (2015) Study of leptin and adiponectin as disease markers in subjects with obstructive sleep apnea. Dis Markers 2014(1):706314

39. Inamoto Y, Saitoh E, Okada S, Kagaya H, Shibata S, Baba M, Onogi K, Hashimoto S, Katada K, Wattanapan P (2015) Anatomy of the larynx and pharynx: effects of age, gender and height revealed by multidetector computed tomography. J Oral Rehabil 42(9):670-677

40. H J, S H, T M, F T, T H, T K, O N (1998) Pharyngeal crosssectional area and pharyngeal compliance in normal males and females. Respiration 65(6):458-468

41. Gun JD, Young CH, Grunstein RR et al (2004) Predictive value of Kushida index and acoustic pharyngometry for the evaluation of upper airway in subjects with or without obstructive sleep apnea [J]. Journal of Korean Medicalence 19(5):662-667

42. Deyoung PN, Bakker JP, Sands SA, Batool-Anwar S, Connolly JG, Butler JP, Malhotra A (2013) Acoustic pharyngometry measurement of minimal cross-sectional airway area is a significant independent predictor of moderate-to-severe obstructive sleep apnea [J]. Journal of clinical sleep medicine: JCSM : official publication of the American Academy of Sleep Medicine 9(11):1161-1164

Publisher's note Springer Nature remains neutral with regard to jurisdictional claims in published maps and institutional affiliations. 